\title{
Short communication: Cold atmospheric plasma inactivation of Prototheca zopfii isolated from bovine milk
}

\author{
E. Tyczkowska-Sieron, ${ }^{* 1}$ J. Markiewicz,† B. Grzesiak, ${ }^{*}$ H. Krukowski, $\ddagger$ A. Glowacka, ${ }^{*}$ and J. Tyczkowski†§ \\ *Department of Environmental Biology, Medical University of Lodz, Zeligowskiego 7/9, 90-752 Lodz, Poland \\ †Department of Molecular Engineering, Faculty of Process and Environmental Engineering, Lodz University of Technology, Wolczanska 213, \\ 90-924 Lodz, Poland \\ ‡Department of Animal and Environmental Hygiene, University of Life Sciences, Akademicka 13, 20-950 Lublin, Poland \\ §Research Institute of Electronics, Shizuoka University, 3-5-1 Johoku, Naka-ku, Hamamatsu, 432-8011 Japan
}

\section{ABSTRACT}

Mastitis is a serious bovine diseases that can be caused by Prototheca zopfii, yeast-like algae belonging to the family Chlorellaceae. The substantial economic losses and health damage associated with bovine mastitis emphasize the need to develop effective strategies aimed at control of the infection. Unfortunately, $P$. zopfii is highly resistant to most common antibacterial and antifungal agents, as well as to heat treatment. We report here the first attempt to use cold atmospheric plasma to inactivate this pathogen. We studied 20 strains of $P$. zopfii isolated from milk samples taken from cows with clinical or subclinical mastitis. The studies confirmed the high level of resistance of $P$. zopfii to typical antifungal agents, such as voriconazole, fluconazole, amphotericin B, caspofungin, anidulafungin, and micafungin. In contrast, each of the strains revealed high susceptibility to cold atmospheric plasma, $>2$-fold higher compared with a reference strain of Candida albicans. The obtained results are promising and open up a new approach in the fight against $P$. zopfii.

Key words: Prototheca zopfii, bovine mastitis, cold atmospheric plasma, microorganism inactivation

\section{Short Communication}

Prototheca zopfii is a chlorophyllous algae belonging to the family Chlorellaceae, and it is a major cause of mastitis in dairy cows (Jánosi et al., 2001; Möller et al., 2007; Jagielski et al., 2011; Sobukawa et al., 2012). Mastitis contributes to large financial losses by dairy farmers because it substantially reduces the production and quality of milk (Milanov et al., 2016). The difficulty in treating cows is the resistance of $P$. zopfii to

Received May 31, 2017.

Accepted September 4, 2017.

${ }^{1}$ Corresponding author: ewa.tyczkowska-sieron@umed.lodz.pl a majority of commonly used antibacterial and antifungal agents (Marques at al., 2006; Jagielski et al., 2012), which is attributed to the presence of sporopollenin in its cell walls (Atkinson at al., 1972; Puel et al., 1987). Sporopollenin is likely also responsible for the high heat resistance of $P$. zopfii, which lowers the effectiveness of milk pasteurization and poses a threat to public health (Melville et al., 1999; Marques et al., 2010; Lassa et al., 2011). In this situation, new methods are sought to effectively fight this problem. For example, Bouari et al. (2014) proposed the use of natural preparations, such as essential oils, and Salerno et al. (2010) evaluated the in vitro algicidal effect of sodium hypochlorite and iodine-based antiseptics on P. zopfii.

Another new method, which we report in this paper, is the cold atmospheric plasma technique that has potential for both milk decontamination (Gurol et al., 2012) and direct treatment of cows affected by protothecal mastitis. This technique is increasingly used in human medicine (Fridman and Friedman, 2013) and food technology (Niemira, 2012). Recently, interest has grown in using the method in veterinary medicine (Niedziela, 2014; Hung et al., 2016). Cold plasma offers a valid replacement of traditional chemical-based agents. Its application in the inactivation of pathogens, in particular, avoids the recurrent problem of drug-resistant microorganisms (Laroussi et al., 2012; Shintani and Sakudo, 2016).

The investigated material consisted of 20 strains of $P$. zopfii that were isolated from milk samples taken in Opole province (Poland) from cows with clinical or subclinical mastitis. To identify this pathogen, the samples were cultured on Sabouraud dextrose chloramphenicol agar (bioMérieux, Marcy l'Etoile, France), incubated for $48 \mathrm{~h}$ at $35^{\circ} \mathrm{C}$, and then Gram stained. Identification was performed using routine cultures, macro- and microscopic morphological characterization, and API 20CAUX (bioMérieux) methods (Padhye et al., 1979). To confirm the accuracy of the strains' classification as $P$. zopfii, clotrimazole susceptibility testing using 
antibiotic discs (50 $\mu \mathrm{g}$ of clotrimazole) was performed (Casal and Gutierrez, 1983).

The in vitro activity of typical antifungal agents (voriconazole, fluconazole, amphotericin B, caspofungin, anidulafungin, and micafungin) was determined using the Etest method (bioMérieux). Prototheca zopfii inoculum suspensions were prepared in sterile saline $(0.85 \% \mathrm{NaCl})$ with an optical density of McFarland standard scale 0.5 (approximately $5 \times 10^{6} \mathrm{cfu} / \mathrm{mL}$ ). The cell density was adjusted with a McFarland Desintometer (type DEN-1B, Biosan, Riga, Latvia). The suspensions were directly inoculated on plates with RPMI-1640 agar, which served as the base medium, onto which test strips (BioMérieux) were placed. The agar plates were incubated at $35^{\circ} \mathrm{C}$ and read after 48 $\mathrm{h}$. The MIC was the lowest concentration at which the border of the elliptical zone of growth inhibition intersected the scale on the test strip. The comparative test for the reference strain of Candida albicans (ATCC 10231) was conducted in the same manner. The results of the susceptibility tests are summarized in Table 1.

As a source of plasma for the cold atmospheric plasma treatment, we used a linear microdischarge jet (Tyczkowski et al., 2012). A schematic view of the experimental system and the working plasma jet are shown in Figure 1a. Plasma was generated at 13.56 $\mathrm{MHz}$ using $\mathrm{He}$ as the reactive gas. The gas flow rate and the discharge power were $1.9 \mathrm{~L} / \mathrm{min}$ and $17 \mathrm{~W}$, respectively. The plasma beam was cylindrical with a length of $4 \mathrm{~cm}$ and a diameter of about $1.5 \mathrm{~mm}$ (Figure 1a), and was fixed parallel to the treated surface of a culture at a distance of $5 \mathrm{~mm}$. Each culture was prepared by uniformly spreading $100 \mu \mathrm{L}$ of PBS containing $5 \times 10^{7} \mathrm{cfu} / \mathrm{mL}$ ( 5 units of the McFarland scale) on the plates with Sabouraud dextrose agar (BioMérieux). The cultures were exposed to the plasma at various times ranging from 1 to $12 \mathrm{~min}$. Then, the plates were incubated at $35^{\circ} \mathrm{C}$ for $24 \mathrm{~h}$.

The growth inhibition zone induced by plasma treatment was elliptical, such that the major axis was parallel to the plasma beam (Figure 1b). The size of the inhibition zone (assumed to be half of the minor axis, c) was determined by densitometric analysis of photographs of the plates, which was performed using Scion Image for Windows software (release alpha 4.0.3.2; Scion Corp., Frederick, MD). The zone edge was defined as the line where viability of the cells reached $10 \%$ survival in the nontreated plasma region. Each measurement was repeated 5 times and the result was averaged. The measurement error did not exceed $5 \%$.

An example of the dependence of inhibition zone size on plasma treatment time is shown for strain XIV in Figure 2. These results are successfully fitted $(P=$ $\left.0.00005, R^{2}=0.988\right)$ by a theoretical curve plotted ac-

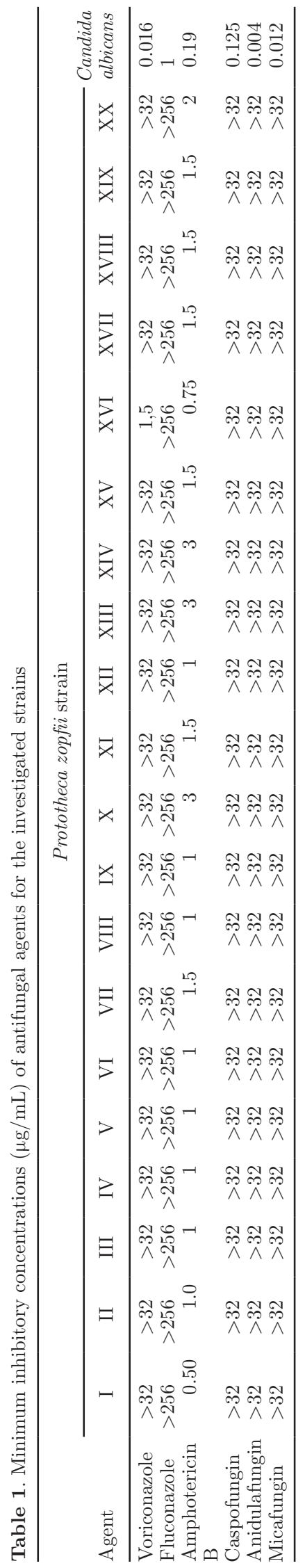

Journal of Dairy Science Vol. 101 No. 1, 2018 
(a)

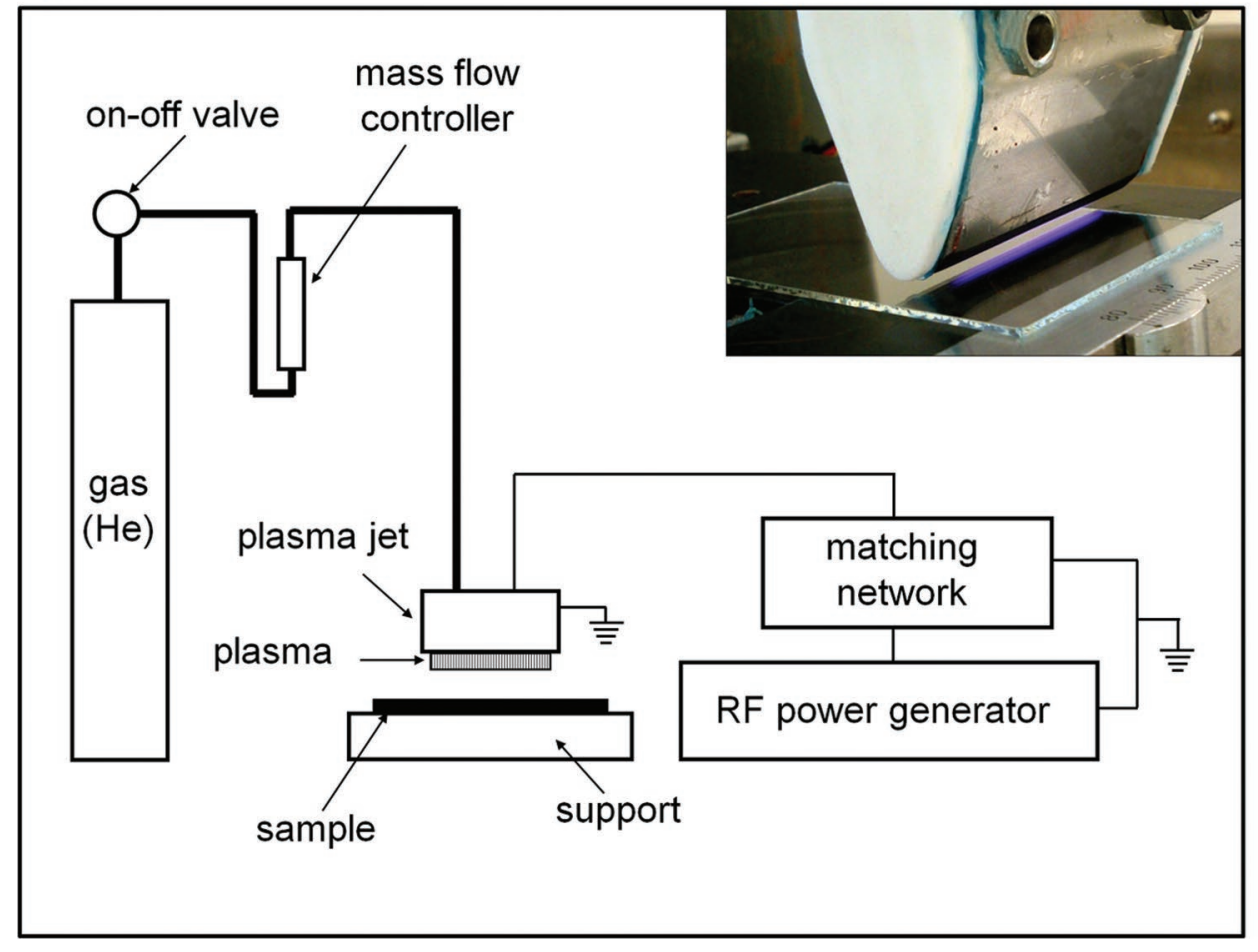

(b)

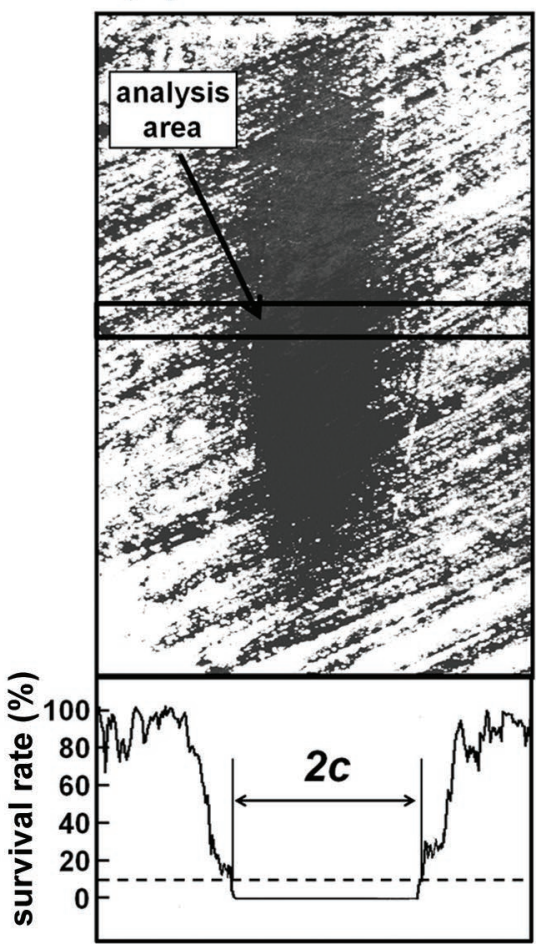

Figure 1. Cold plasma treatment: (a) schematic view of the experimental system ( $\mathrm{RF}=$ radio-frequency); inset photograph shows the working linear microdischarge jet; and (b) an example of densitometric analysis determining the size of inhibition zone, c, of Prototheca zopfii growth under the influence of plasma. Color version available online.

cording to Eq. [1], which was derived on the basis of a simple model describing the spread of killing agents (e.g., free radicals, ions, UV photons, excited molecules) from the plasma center (Tyczkowska-Sieroń and Markiewicz, 2014):

$$
\left.c=\left(\alpha d t / D_{\min }\right)^{2 / 3}-d^{2}\right)^{1 / 2}
$$

where $c$ is the size of the inhibition zone (defined in Figure $1 \mathrm{~b}), \alpha$ is a parameter related to the concentration of killing agents in the plasma center (in this experiment, $\alpha$ is constant because the conditions of plasma generation were constant), $d$ is the distance from the center of the plasma beam measured perpendicularly to the treated surface of the culture, $t$ is the plasma treatment time, and $D_{\min }$ is the minimum lethal dose of plasma (90\% killing of the cell population). The fitting was carried out using the software of TableCurve 1.12 (Jandel Scientific Inc., San Rafael, CA).

From the fitted function (Eq. [1]), the $D_{\min }$ value can be calculated. In the case of the strain XIV, this value was 2.61 (arbitrary units). Analogous analysis of the dependence of $c$ versus $t$ was carried out for all other $P$. zopfii isolates. The values of $D_{\min }$ calculated on this basis are presented in Figure 3. For comparison, we show the $D_{\min }$ value for the reference strain of Candida albicans subjected to plasma action in the same experimental system and under the same conditions as the $P$. zopfii strains.

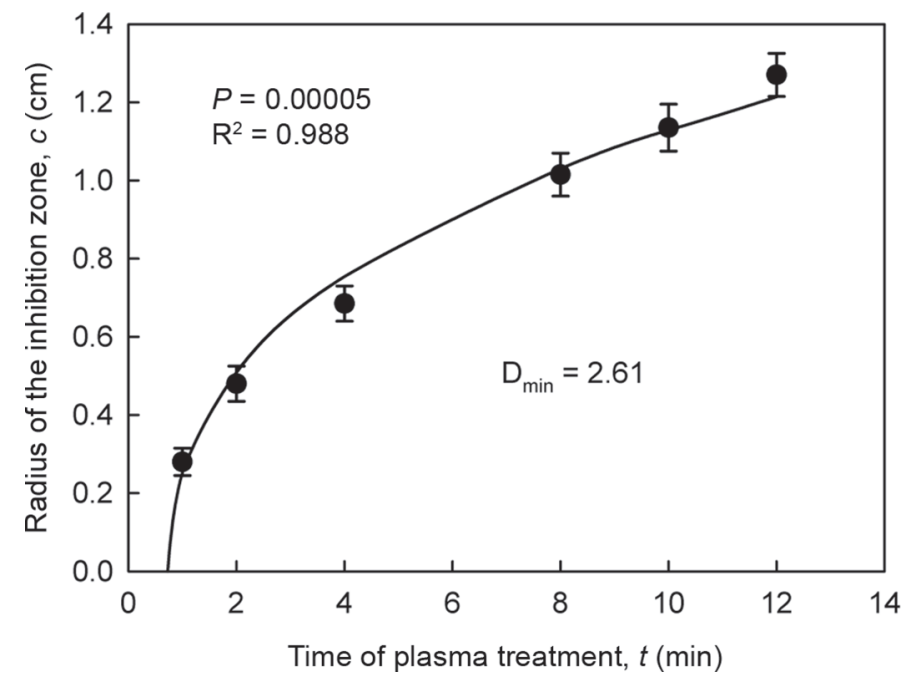

Figure 2. Dependence of the size of inhibition zone, c, of Prototheca zopfii growth on plasma treatment time, $t(\bullet) . D_{\min }=$ minimum lethal dose (arbitrary units). The curve represents the fit of the experimental points according to Eq. [1]. Error bars represent SE. 


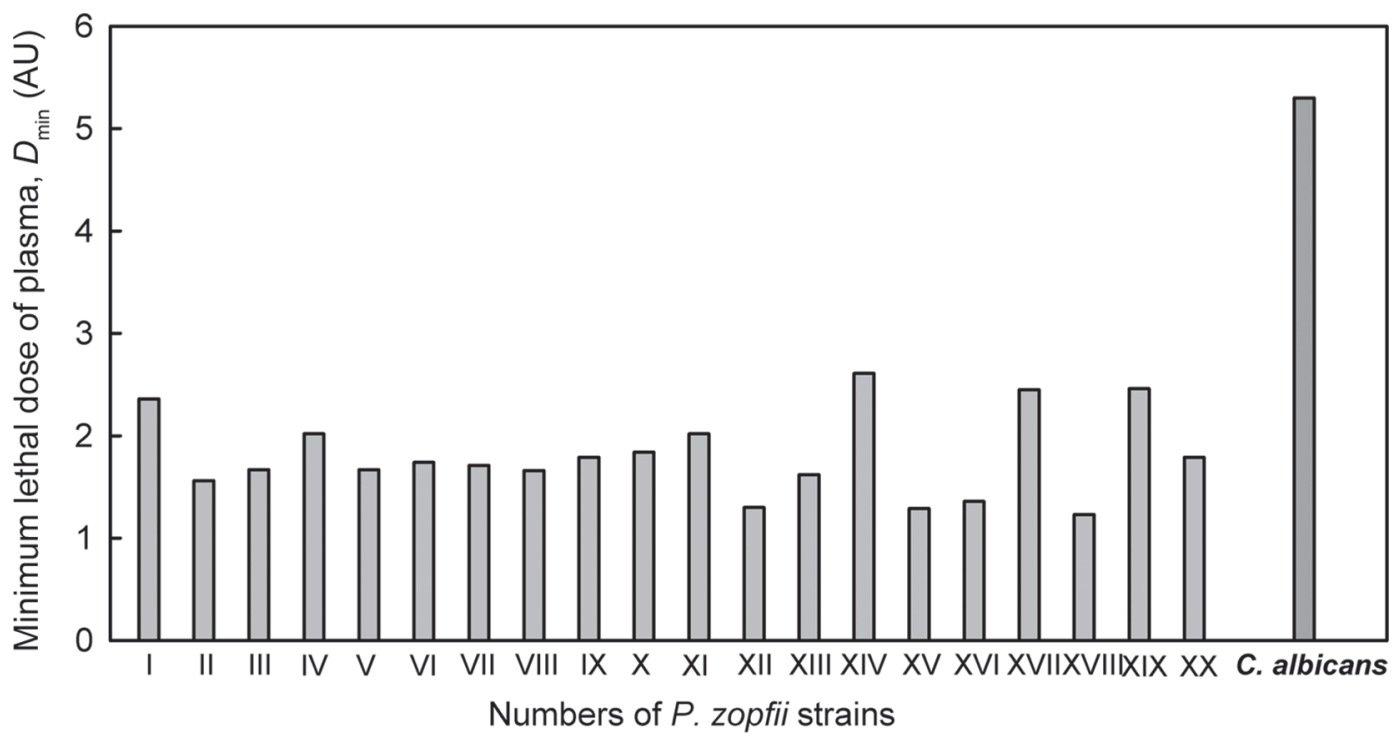

Figure 3. Minimum lethal dose (arbitrary units) of plasma $\left(D_{\min }\right)$ calculated for the investigated strains of Prototheca zopfii and Candida albicans (reference strain).

The results of susceptibility testing of the isolated $P$. zopfii strains confirmed the consensus that eradication of this pathogen is a serious problem (Marques at al., 2006; Jagielski et al., 2012). As can be seen in Table 1, only amphotericin B showed some efficacy against all the tested strains of $P$. zopfii. For the other agents, $P$. zopfii was completely resistant. In contrast, our investigations using the cold plasma method demonstrated its significant killing effect, which was similar for all $P$. zopfii isolates. It was surprising, however, that $P$. zopfii strains were considerably more sensitive to the plasma treatment (lower values of $D_{\min }$ ) than C.albicans, despite the fact that the $P$. zopfii strains are much more resistant to antifungal drugs (Table 1). This indicates a completely different mechanism of action of the plasma method on microorganisms compared with conventional antifungal agents.

At this initial stage of research, it is difficult to determine precisely the killing mechanism of the tested method. However, our previous studies (E. TyczkowskaSieron, unpublished data) have proved that this process must be closely related to the direct interaction between the cells and plasma species, such as free radicals, ions, UV photons, or reactive molecules. The operation with He flux only (i.e., without plasma generation) under the same conditions as the plasma treatment and that using electric field alone (voltage applied to electrodes without the He flux; i.e., without plasma generation) did not produce any biological effects. Also, no effects were observed when culturing the pathogens on medium previously plasma treated under the conditions used in our research. The possibility of a thermal effect must also be rejected. We measured the surface temperature of the plated medium using a thermocouple under the conditions of our plasma treatment and showed that the temperature increased by only $1^{\circ} \mathrm{C}$ (from $17^{\circ} \mathrm{C}$ to $18^{\circ} \mathrm{C}$ ) for the first 6 min and was then stable over time.

Further research to determine the main plasma agents responsible for the process of $P$. zopfii killing is necessary. The primary candidates are UV photons and free radicals, based on oxygen and nitrogen drawn into the plasma region from air (Poiata et al., 2010). However, we can already conclude that the cold plasma treatment is very effective in eradicating $P$. zopfii cells.

The results presented in this paper are promising but it is necessary to undertake further studies on the use of cold plasma for the decontamination of $P$. zopfii in milk and as a supportive therapy for the treatment of cows affected by protothecal mastitis. The drastic difference in susceptibility to plasma treatment between $P$. zopfii and $C$. albicans is another interesting area for further research on the mechanism of interaction between cold plasma and microorganisms.

\section{ACKNOWLEDGMENTS}

This work was supported by Ministry of Science and Higher Education of the Republic of Poland (statute investigations).

\section{REFERENCES}

Atkinson, A. W., B. E. S. Gunning, and P. C. L. John. 1972. Sporopollenin in the cell wall of Chlorella and other algae: Ultrastructure, 
chemistry, and incorporation of $14 \mathrm{C}$-acetate, studied in synchronous cultures. Planta 107:1-32.

Bouari, C., P. Bolfa, G. Borz, G. Nadăş, C. Cătoi, and N. Fiţ. 2014. Antimicrobial activity of Mentha piperita and Saturenja hortensis in a murine model of cutaneous protothecosis. J. Mycol. Med. $24: 34-43$.

Casal, M. J., and J. Gutierrez. 1983. Simple new test for rapid differentiation of Prototheca wickerhamii from Prototheca zopfii. J. Clin. Microbiol. 18:992-993.

Fridman, A., and G. Friedman. 2013. Plasma Medicine. John Wiley \& Sons Ltd., Chichester, UK.

Gurol, C., F. Y. Ekinci, N. Aslan, and M. Korachi. 2012. Low temperature plasma for decontamination of E. coli in milk. Int. J. Food Microbiol. 157:1-5.

Hung, Y. W., L. T. Lee, Y. C. Peng, C. T. Chang, Y. K. Wong, and K. C. Tung. 2016. Effect of a nonthermal-atmospheric pressure plasma jest on wound healing: An animal study. J. Chin. Med. Assoc. 79:320-328.

Jagielski, T., P. Buzzini, H. Lassa, E. Malinowski, E. Branda, B. Turchetti, A. Polleichtner, U. Roesler, P. E. Lagneau, S. Marques, E. Silva, G. Thompson, R. Stachowiak, and J. Bielecki. 2012. Multicentre Etest evaluation of in vitro activity of conventional antifungal drugs against European bovine mastitis Prototheca spp. isolates. J. Antimicrob. Chemother. 67:1945-1947.

Jagielski, T., H. Lassa, J. Ahrholdt, E. Malinowski, and U. Roeslerc. 2011. Genotyping of bovine Prototheca mastitis isolates from Poland. Vet. Microbiol. 149:283-287.

Jánosi, S., F. Ratz, G. Szigeti, M. Kulcsar, J. Kerényi, T. Laukó, F. Katona, and G. Huszenicza. 2001. Pathophysiology: Review of the microbiological, pathological, and clinical aspects of bovine mastitis caused by the alga Prototheca zopfii. Vet. Q. 23:58-61.

Laroussi, M., M. G. Kong, G. Morfill, and W. Stolz. 2012. Plasma Medicine: Applications of Low-Temperature Gas Plasmas in Medicine and Biology. Cambridge University Press, Cambridge, UK.

Lassa, H., T. Jagielski, and E. Malinowski. 2011. Effect of different heat treatments and disinfectants on the survival of Prototheca zopfii. Mycopathologia 171:177-182.

Marques, S., E. Silva, J. Calvalheira, and G. Thompson. 2006. In vitro antimicrobial susceptibility of Prototheca wickerhamii and Prototheca zophii from bovine mastitis. J. Dairy Sci. 89:4202-4204.

Marques, S., E. Silva, J. Calvalheira, and G. Thompson. 2010. Temperature sensibility of Prototheca blaschkeae strains isolated from bovine mastitic milk. J. Dairy Sci. 93:5110-5113.

Melville, P. A., E. T. Watanabe, N. R. Benites, A. R. Ribeiro, J. A. Silva, F. Garino Junior, and E. O. Costa. 1999. Evaluation of the susceptibility of Prototheca zopfii to milk pasteurization. Mycopathologia 146:79-82.

Milanov, D., T. Petrović, V. Polaček, L. Suvajdžić, and J. Bojkovski. 2016. Mastitis associated with Prototheca zopfii - an emerging health and economic problem on dairy farms. J. Vet. Res. 60:373-378.

Möller, A., U. Truyen, and U. Roesler. 2007. Prototheca zopfii genotype 2: The causative agent of bovine protothecal mastitis? Vet. Microbiol. 120:370-374.

Niedziela, K. 2014. Is plasma the future of veterinary medicine? Veterinary Practice News. http://www.veterinarypracticenews.com/Is -Plasma-The-Future-Of-Veterinary-Medicine/.

Niemira, B. A. 2012. Cold plasma decontamination of foods. Annu. Rev. Food Sci. Technol. 3:125-142.

Padhye, A. A., J. G. Baker, and D. F. D'Amato. 1979. Rapid identification of Prototheca species by the API 20C System. J. Clin. Microbiol. 10:579-582.

Poiata, A., J. Montrescu, A. Nastuta, D. E. Creanga, and G. Popa 2010. Microorganism response to atmospheric pressure helium plasma DBD treatment. J. Electrost. 68:128-131.

Puel, F., C. Largeau, and G. Giraud. 1987. Occurrence of a resistant biopolymer in the outer walls of the parasitic alga Prototheca wickerhamii (Chlorococcalses): Ultrastructural and chemical studies. J. Phycol. 23:649-656.

Salerno, T., M. G. Ribeiro, H. Langoni, A. Keller Siqueira, E. Oliveira da Costa, P. A. Melville, V. Ferreira, F. Bueno, A. A. M. Yamamura, U. Roesler, and A. V. da Silva. 2010. In vitro algaecide effect of sodium hypochlorite and iodine based antiseptics on Prototheca zopfii strains isolated from bovine milk. Res. Vet. Sci. 88:211-213.

Shintani, H., and A. Sakudo. 2016. Gas Plasma Sterilization in Microbiology: Theory, Applications, Pitfalls and New Perspectives. Caister Academic Press, Norfolk, UK

Sobukawa, H., S. Yamaguchi, R. Kano, T. Ito, K. Suzuki, M. Onazaki, A. Hasegawa, and H. Kamata. 2012. Molecular typing of Prototheca zophii from bovine mastitis in Japan. J. Dairy Sci. 95:4442-4446.

Tyczkowska-Sieroń, E., and J. Markiewicz. 2014. Inactivation of Candida species using cold atmospheric plasma on the way to a new method of eradication of superficial fungal infections. Med. Dosw. Mikrobiol. 66:121-129.

Tyczkowski, J., P. Kazimierski, and J. Zieliński. 2012. Microplasma electrode reactor for the surface treatment under atmospheric pressure. Polish Pat. No. 212569 